\title{
Identification and Analysis of Genes Involved in the Jasmonate Pathway in Response to Ethephon and 1-Methylcyclopropene during the Ripening of Apple Fruit
}

\author{
Jingyi Lv' ${ }^{\mathbf{1}}$, Yonghong Ge, Canying Li, Mengyuan Zhang, and Jianrong Li ${ }^{\mathbf{1}}$ \\ College of Food Science and Project Engineering, Bohai University, Jinzhou, Liaoning 121013, PR \\ China; and National \& Local Joint Engineering Research Center of Storage, Processing and Safety \\ Control Technology for Fresh Agricultural and Aquatic Products, Bohai University, Jinzhou, \\ Liaoning 121013, PR China
}

\begin{abstract}
AdDitional INDEX WORDs. Malus domestica, expression analysis, plant hormones, signal transduction
ABstract. Fruit ripening is a complex process involving many physiological changes and the dynamic interplay between different phytohormones. In addition to ethylene, jasmonates (JAs) have also been demonstrated to play an important role in the regulation of fruit ripening. However, the mechanisms underlying the interaction between these two pathways during fruit ripening are unknown. In recent years, research has been conducted to illustrate the effects of JAs on the ethylene biosynthesis and signaling pathway, but little is known regarding the effects of ethylene on JA biosynthesis and the signaling pathway during fruit ripening. Herein, we aimed to evaluate the effects of ethylene on JA biosynthesis in ripening apple (Malus $\times$ domestica) fruit and on the expression of key genes involved in the JA biosynthesis and the signaling pathway. For this purpose, we treated apple fruit with ethephon and 1-methylcyclopropene (1-MCP) at commercial maturity. Our data indicated that endogenous JA content and allene oxide synthase (AOS) activity were reduced by ethephon treatment at the early ripening stage, whereas they were enhanced by 1-MCP treatment at the late ripening stage. Quantitative real-time polymerase chain reaction (PCR) analysis revealed that the expression profiles of three $A O S$ genes (MdAOS2, MdAOS3, and MdAOS5) and two lipoxygenase (LOX) genes (MdLOX22 and MdLOX28) showed similar trends with the change of AOS activity in all groups during fruit ripening. The expression of $M d L O X 21$ and $M d L O X 23$ was in accordance with the change of ethylene production on ripening, and it was positively regulated by ethylene, whereas the opposite effect was observed for MdLOX39 expression. The transcription of MdLOX310 and MdLOX61 appeared unaffected by ethylene during fruit ripening. Three jasmonate ZIM-domain (JAZ) genes (MdJAZ9, MdJAZ10, and $M d J A Z 18$ ) were differentially upregulated by ethephon treatment whereas being downregulated by 1-MCP treatment during fruit ripening. Expression of $M d J A Z 13$ and $M d J A Z 14$ was downregulated at the early ripening stage by both treatments. Our results suggested regulating roles of ethylene on the JA biosynthesis and signaling pathway during fruit ripening and senescence.
\end{abstract}

Apple fruit is a climacteric fruit, which is characterized by an exponential increase in ethylene production and respiration rate, as well as changes in fruit color, texture, and aroma during ripening (Yang and Hoffman, 1984). Ethylene has been identified as the major hormone that initiates and controls ripening in fleshy fruit for longer than a decade (Liu et al., 2015). However, fruit ripening is a genetically programmed process that is coordinated by a complex network of endogenous hormones. In addition to ethylene, JAs have also been demonstrated to play an important role in the regulation of the fruit maturation and ripening process (Kondo, 2010). Many studies have confirmed that exogenous JA application can promote volatile emission (Kondo et al., 2005) and stimulate carotene synthesis (Pérez et al., 1993) and anthocyanin accumulation (Rudell and Mattheis, 2008).

The term jasmonates often is used to refer to jasmonic acid, its derivatives, and conjugates (Wasternack, 2007). These molecules are ubiquitous lipid-derived signaling compounds in plants, and

Received for publication 24 Jan. 2017. Accepted for publication 17 Mar. 2017. This work was supported by the National Natural Science Foundation of China (grant numbers 31501537 and 31401554) and the Doctor Startup Fund Program of Bohai University (grant number 0515bs033-1).

'Corresponding authors. E-mail: jingyi.lv@foxmail.com or 1ijr6491@163.com. they play essential roles in plant growth, development, and defense (Katsir et al., 2008). Biosynthesis of JA begins with $\alpha$-linolenic acid, which is oxygenated by 13-lipoxygenase (13-LOX), and the resulting compound is dehydrated by AOS and subsequently proceeds through a series of reactions to produce jasmonic acid, which can be further converted into its derivatives or conjugates such as methyl-JA (MeJA) and jasmonyl-isoleucine (JA-Ile) (Wasternack, 2007). AOS is the first specific enzyme and the major control point of the JA biosynthesis pathway (Laudert and Weiler, 1998). JA initiates its signaling process on the formation of a $\mathrm{SCF}^{\mathrm{COI}}$-JA-Ile-JAZ [Skp/Cullin/F-box (SCF), coronatine insensitive 1 (COI1), jasmonate ZIM domain (JAZ)] ternary complex, in which the JAZ repressors are ubiquitinated and subsequently degraded to release transcription factors (TFs), leading to downstream gene expression (Thines et al., 2007). Recent discoveries showed that JAZ transcriptional repressors are key regulators of JA signaling and that they function as crucial interfaces to mediate the synergy and antagonism between JA and other phytohormones, such as ethylene and gibberellin (Song et al., 2014).

Indeed, the cross talk between the JA and ethylene pathways in regulating plant developmental and defense-related processes has been well documented in recent years (Pieterse et al., 2009). 
However, the interactions between the two hormones in regulating fruit ripening and senescence are unclear. In the past few years, studies have mainly focused on the role of JAs on the ethylene pathway during fruit ripening. In climacteric fruit, such as apples (Kondo et al., 2009), pears [Pyrus communis (Kondo et al., 2007)], and peaches [Prunus persica (Ruiz et al., 2013)], exogenous JA application modulated ethylene biosynthesis and expression of its biosynthesis genes in a developmental- and concentration-dependent fashion. However, to date, few studies have reported the effects of ethylene on the JA biosynthesis and signaling pathway during fruit ripening. Only studies in kiwifruit (Actinidia deliciosa) showed that the peak of AOS activity was significantly delayed by 1 -MCP treatment, whereas it was induced by ethephon treatment during fruit ripening (Ding et al., 2003). In arabidopsis (Arabidopsis thaliana), ethylene signaling can inhibit JA-dependent responses (Ellis and Turner, 2001) and suppress the JA pathway in ozone-induced spreading cell death (Tuominen et al., 2004). Whether ethylene has the same effects on the JA pathway during fruit ripening deserves careful investigation, especially at the molecular level.

1-Methylcyclopropene, a powerful ethylene antagonist, is thought to bind to ethylene receptors and, thereby, effectively blocks the ethylene signal transduction pathway from the first step (Watkins, 2006). Ethephon is an ethylene-yielding chemical that, when applied directly to plants, can elicit a response, which is a characteristic of ethylene treatment. The aim of this study was to investigate the hypothesis that ethylene might regulate the JA biosynthesis and signaling pathway during the ripening of apple fruit by applying 1-MCP and ethephon.

\section{Materials and Methods}

Plant materials and treatments. 'Golden Delicious' apple fruit without mechanical damage and free of visible defects or decay were harvested at commercial maturity from a commercial orchard in Jinzhou, China. Fruit were randomly sampled from over 80 individual trees and immediately transported to the postharvest laboratory of Bohai University. The selected fruit were randomly divided into three groups, with 180 fruit per group. Each group was then divided into three subgroups, and one subgroup of 60 fruit was selected for control, ethephon, or 1-MCP treatment. Ethephon treatment was performed by immersing fruit into $0.2 \mathrm{mmol} \cdot \mathrm{L}^{-1}$ ethephon (Solarbio, Beijing, China) for $20 \mathrm{~min}$. 1-MCP treatment was performed by enclosing fruit in $125-\mathrm{L}$ plastic containers with $1 \mu \mathrm{L} \cdot \mathrm{L}^{-1} 1-\mathrm{MCP}$ (Fresh doctor; Xiqin Biotechnology, Xianyang, China) for $24 \mathrm{~h}$. Fruit were placed in the container, treatment was given with a small portable ventilation fan (F95; Bei Yinmei Electronics Technology, Shenzhen, China) for uniform mixing and distribution of 1-MCP, a plastic flask containing $1.5332 \mathrm{~g}$ of $0.018 \%$ 1-MCP powder was placed inside the container, warm water (around $30^{\circ} \mathrm{C}$ ) was quickly poured into the plastic flask (ratio of 1-MCP:water $=1: 40$ ), and then the container was immediately sealed for $24 \mathrm{~h}$. Fruit of nontreatment was served as the control. All treated and control fruit were stored in open cardboard boxes and placed in the laboratory at $25^{\circ} \mathrm{C}$. Fruit were sampled at $0,7,14,21,28$, and $35 \mathrm{~d}$. At each sampling time, a total of 12 fruit were randomly selected from the three treatment subgroups with four fruit for each subgroup for the measurement of ethylene production and fruit firmness, and the other nine fruit with three fruit for each subgroup were pooled together (cortex with skin), frozen in liquid nitrogen, and stored immediately at $-80{ }^{\circ} \mathrm{C}$ until further use.

ETHYLENE PRODUCTION, FLESH FIRMNESS, AND STARCH-IODINE INDEX. Ethylene production was determined by enclosing four apples in sealed 9.35-L glass desiccators for $1 \mathrm{~h}$ at room temperature, and then a 1-mL sample of the headspace gas was collected with a syringe and injected into a flame ionization detection gas chromatograph (GC-14A; Shimadzu, Kyoto, Japan) fitted with a flame-ionization detector and an activated alumina column $(200 \mathrm{~cm} \times 0.3 \mathrm{~cm})$. The oven, detector, and injector were operated at 70,70 , and $150{ }^{\circ} \mathrm{C}$, respectively. The carrier gas $\left(\mathrm{N}_{2}, \mathrm{H}_{2}\right.$, and air) flow rates were $0.5,0.5$, and $5 \mathrm{~mL} \cdot \mathrm{s}^{-1}$, respectively. Three biological replicates were included for each treatment, and ethylene concentrations were measured three times for each replicate. The rate of ethylene production was expressed as micromoles per kilogram per second.

Flesh firmness was determined at each time point from 12 fruit after removing a small disk of skin and then measuring each fruit three times from three positions located at $120^{\circ}$ intervals around the equator. Measurements were conducted with a handheld pressure tester (model FT327; Effegi, Milan, Italy) equipped with a 5-mm-diameter probe and expressed in Newton.

The starch-iodine index was visually rated using the Cornell generic starch scale 1-8 (Blanpied and Silsby, 1992). Apples were cut in half at the equator, dipped in potassium iodine solution, and rated on a scale of $1-8$, with 1 representing no starch degradation and 8 representing complete degradation. The starch-iodine index was calculated by averaging the values of 12 apples.

AnAlysis of JA CONTENT AND AOS ACTIVITy. The JA content was measured using conventional indirect competitive enzymelinked immunosorbent assay (icELISA) according to the methods of Deng et al. (2008). Fresh fruit tissue $(0.5 \mathrm{~g})$ was used for the preparation of the extracts at each time point, and the content of JAs was expressed as micrograms per kilogram on fresh weight basis. AOS activity was analyzed following the protocol described by Zimmerman and Vick (1970). One unit of AOS activity was defined as the amount of enzyme required to cause the decrease of one absorbance unit at $234 \mathrm{~nm}$ in $1 \mathrm{~min}$. Three biological samples were taken from the pooled tissues for each treatment, and each sample was measured three times.

Total RNA EXTRACTION AND SCREENING OF SPECIFICEXPRESSED GENE FAMILY MEMBERS. Total RNA from each sample was extracted according to the manufacturer's instructions for the RNAprep pure plant kit (Tiangen, Beijing, China). All RNA extracts were treated with DNase I (Tiangen) to remove contamination from genomic DNA. Conventional PCR was used to assess gene expression in the fruit. About the same amount of RNAs from each weekly sample was pooled. Firststrand cDNA was synthesized from $1 \mu \mathrm{g}$ of the total RNA mixtures using reverse transcriptase M-MLV [RNase H- (Takara, Dalian, China)] and oligo $(\mathrm{dT})_{18}$ as the primer. Based on the positional specificity of the dioxygen insertion using linoleic acid as a substrate, the $L O X$ gene family can be divided into 9-LOX and 13-LOX. Only the 13-LOX genes are believed to participate in JA biosynthesis (Feussner and Wasternack, 2002), so those belonging to the 9-LOX subclass were excluded. According to our previous study (Lv et al., 2015), $22 L O X$ genes and five $A O S$ genes were subjected to screening for their tissue-specific expression after harvest. Seven $J A Z$ genes, which were confirmed to be expressed in apple fruit tissue ( $\mathrm{Li}$ et al., 2015), were selected to verify expression during ripening. The primers for the $L O X$ and $A O S$ 
genes were as described in Lv et al. (2015), and the primers for the $J A Z$ genes were as described in $\mathrm{Li}$ et al. (2015), respectively. Sequences of primers used in this study were shown in Supplemental Table 1. The PCR reactions were performed according to the $2 \times$ Taq PCR Master Mix Kit (Tiangen) in a total volume of $25 \mu \mathrm{L}$ on a PCR system (GeneAmp ${ }^{\circledR} 9700$ PCR System; Applied Biosystems, Foster City, CA). The amplification products were separated on $1 \%$ agarose using gel electrophoresis and analyzed with an ultraviolet transilluminator (Uvitec FireReader; Uvitec, Cambridge, UK).

Real-time Quantitative PCR analysis. The RNA concentration from each weekly sample was measured using a spectrophotometer (NanoDrop ND-1000; Thermo Fisher Scientific, Wilmington, DE). First-strand cDNA was synthesized from 0.5 $\mu \mathrm{g}$ of the total RNA using the PrimeScript RT Master Mix kit (Takara). The cDNA was diluted 10 times. PCR reactions were carried out in a total volume of $20 \mu \mathrm{L}$, including $2 \mu \mathrm{L}$ of the diluted cDNA, $0.4 \mu \mathrm{M}$ of each primer, $0.4 \mu \mathrm{L}$ of ROX reference dye $(50 \times), 6 \mu \mathrm{L}$ of double-distilled water, and $10 \mu \mathrm{L}$ of $2 \times$ SYBR Premix Ex Taq II (Takara) using a real-time PCR detection system (StepOnePlus; Applied Biosystems). The amplification conditions were $30 \mathrm{~s}$ at $95^{\circ} \mathrm{C}$, 40 cycles of $5 \mathrm{~s}$ at $95^{\circ} \mathrm{C}$, and $34 \mathrm{~s}$ at $59{ }^{\circ} \mathrm{C}$. The primers used for all target genes are described in Supplemental Table 1. Mdactin was used as a reference gene according to Guardo et al. (2013). No-template controls for each primer pair were included in each run. The target gene expression was normalized to that of the internal reference gene (Mdactin) according to the $2^{-\Delta \Delta C T}$ method (Livak and Schmittgen, 2001). Samples from day 0 (assigned an arbitrary quantity of " 1 ") were used as the calibrator for calculating the relative expression level of the target gene. The experiment was performed with three biological replicates.

Statistical analysis. Data were tested by using analysis of variance (ANOVA) with SAS software (version 8.0; SAS Institute, Cary, NC). The least significant difference (LSD) values were calculated to compare differences among treatments for each sampling day. Probability values less than 0.05 were considered statistically significant. All measured values were presented as mean $\pm \mathrm{SE}$.

\section{Results}

Physiological Characterizations of Fruit RiPening ANd IN RESPONSE TO ETHEPHON AND 1-MCP TREATMENT. Ethylene production, fruit firmness, and starch-iodine index are ripening indicators of fruit. Ethylene production increased and peaked at $21 \mathrm{~d}$ in the control and ethephon-treated fruit (Fig. 1A). Application of ethephon stimulated ethylene production. The maximum ethylene production $(21 \mathrm{~d})$ in ethephon-treated fruit was 1.2-fold higher than that in controls $(P<0.01)$. In contrast, ethylene production was reduced by 1-MCP treatment from 7 to $28 \mathrm{~d}$, and then it almost recovered
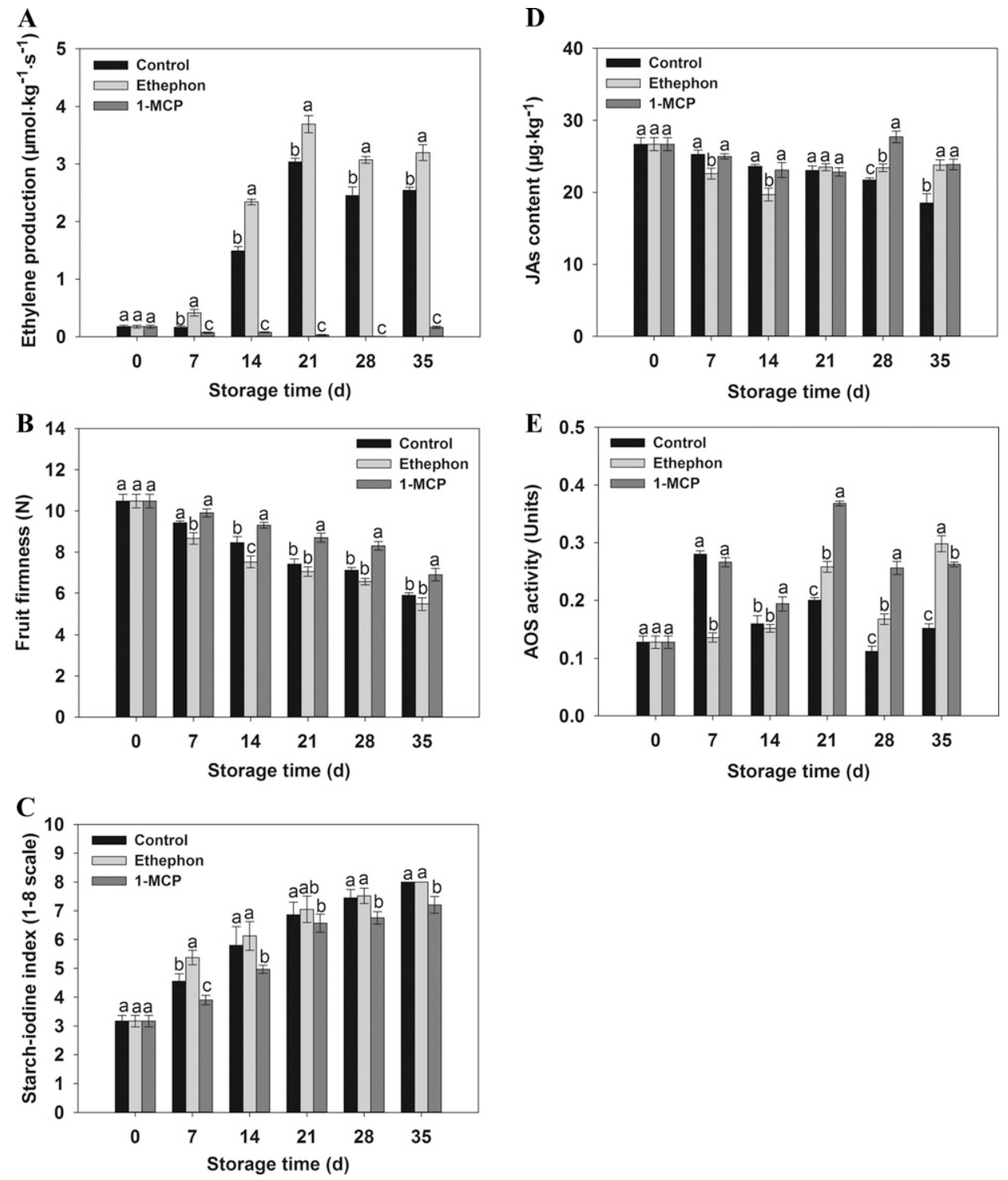

Fig. 1. Physiological indices of apple fruit during storage and after treatment with ethephon and 1-MCP. (A) Ethylene production, (B) fruit firmness, (C) starch-iodine index, (D) JAs content, and (E) AOS activity. Ethephon treatment was performed by immersing fruit into $0.2 \mathrm{mmol} \cdot \mathrm{L}^{-1}$ ethephon for $20 \mathrm{~min}$. 1-MCP treatment was performed by enclosing fruit with $1 \mu \mathrm{L} \cdot \mathrm{L}^{-1} 1-\mathrm{MCP}$ for $24 \mathrm{~h}$. Untreated fruits served as controls. The ethylene production, endogenous JA content, and AOS activity at each time point were calculated from the means of three biological replicates, and each replicate included three technical repeats. Fruit firmness and starch-iodine index were calculated by averaging the values of 12 apples. The starch-iodine index was visually rated using the Cornell generic starch scale 1-8, with 1 representing no starch degradation and 8 representing complete degradation. Vertical bars represent SE. Columns with different letters at each time point indicate significant differences according to LSD test at $P<0.05$. 1-MCP $=1$-methylcyclopropene; JAs $=$ jasmonates; $\mathrm{AOS}=$ allene oxide synthase; LSD $=$ least significant difference. 
to the harvest level at $35 \mathrm{~d}$ when it was 17 -fold higher than the value obtained at $28 \mathrm{~d}$.

Fruit firmness decreased on ripening in all groups (Fig. 1B). Ethephon-treated fruit showed a higher rate of softening than the control fruit from 7 to $14 \mathrm{~d}(P<0.05)$, whereas after $14 \mathrm{~d}$, no significant difference in the firmness value was observed between the two groups. No significant difference in fruit softening between 1-MCP-treated fruit and the control fruit was observed at $7 \mathrm{~d}$, whereas after $7 \mathrm{~d}$, the firmness in 1-MCP-treated fruit was higher than that in the control fruit during storage.

The starch-iodine index showed an increased trend during storage in all groups (Fig. 1C). In ethephontreated fruit, it was higher than the controls at $7 \mathrm{~d}(P<0.05)$, whereas after $7 \mathrm{~d}$, no significant difference was observed between the two groups. In 1-MCP-treated fruit, the slow disappearance of starch was observed; starch-iodine index was lower than the controls during the entire storage period $(P<0.05)$.

ANALYSIS OF JA CONTENT AND AOS ACTIVITY DURING FRUIT RIPENING AND IN RESPONSE TO ETHEPHON AND 1-MCP TREATMENT. The endogenous JA content gradually decreased on ripening in the control fruit (Fig. 1D). In ethephon-treated fruit, the JA content was reduced from 7 to $14 \mathrm{~d}$, and then it increased at $21 \mathrm{~d}$ and showed very little change until the end of storage. The JA content in ethephon-treated fruit was $11 \%$ and $17 \%$ lower than that in the control fruit at 7 and $14 \mathrm{~d}$, respectively. No readily observable difference in JA content was observed between the control and 1-MCP-treated fruit from 7 to $21 \mathrm{~d}$, whereas after $21 \mathrm{~d}$, the JA content in 1-MCP-treated fruit kept higher than that in the control fruit.

In the control fruit, AOS activity peaked at $7 \mathrm{~d}$, and then it declined (Fig. 1E). In the ethephon-treated fruit, AOS activity was reduced at $7 \mathrm{~d}$ when it was only $48.6 \%$ of the level in the control fruit $(P<0.01)$, and then it recovered and remained higher than the controls until the end of storage. In the 1-MCP-treated fruit, AOS activity peaked at $21 \mathrm{~d}$, which was $14 \mathrm{~d}$ later than the controls, and it kept higher than the controls after $21 \mathrm{~d}(P<0.01)$.

Preliminary SCREENING OF APPLE FRUIT-EXPRESSED GENES AFTER HARVEST. Twenty-two LOX genes, five $A O S$ genes, and seven $J A Z$ genes were selected to verify expression using conventional PCR assay. As a result, seven $L O X$ (MdLOX21, MdLOX22, MdLOX23,
$M d L O X 28, M d L O X 39, M d L O X 310$, and MdLOX61), three $A O S$ (MdAOS2, MdAOS3, and MdAOS5), and five JAZ (MdJAZ9, MdJAZ10, MdJAZ13, MdJAZ14, and MdJAZ18) genes were confirmed to be expressed in the ripening apple fruit tissues. Expression patterns of these identified genes were analyzed using the subsequent quantitative real-time PCR assay.

EXPRESSION PATTERNS OF JA BIOSYNTHESIS GENES DURING FRUIT RIPENING AND IN RESPONSE TO ETHEPHON AND 1-MCP TREATMENT. The expression of the $L O X$ genes was differentially regulated by ethephon and 1-MCP treatments during ripening

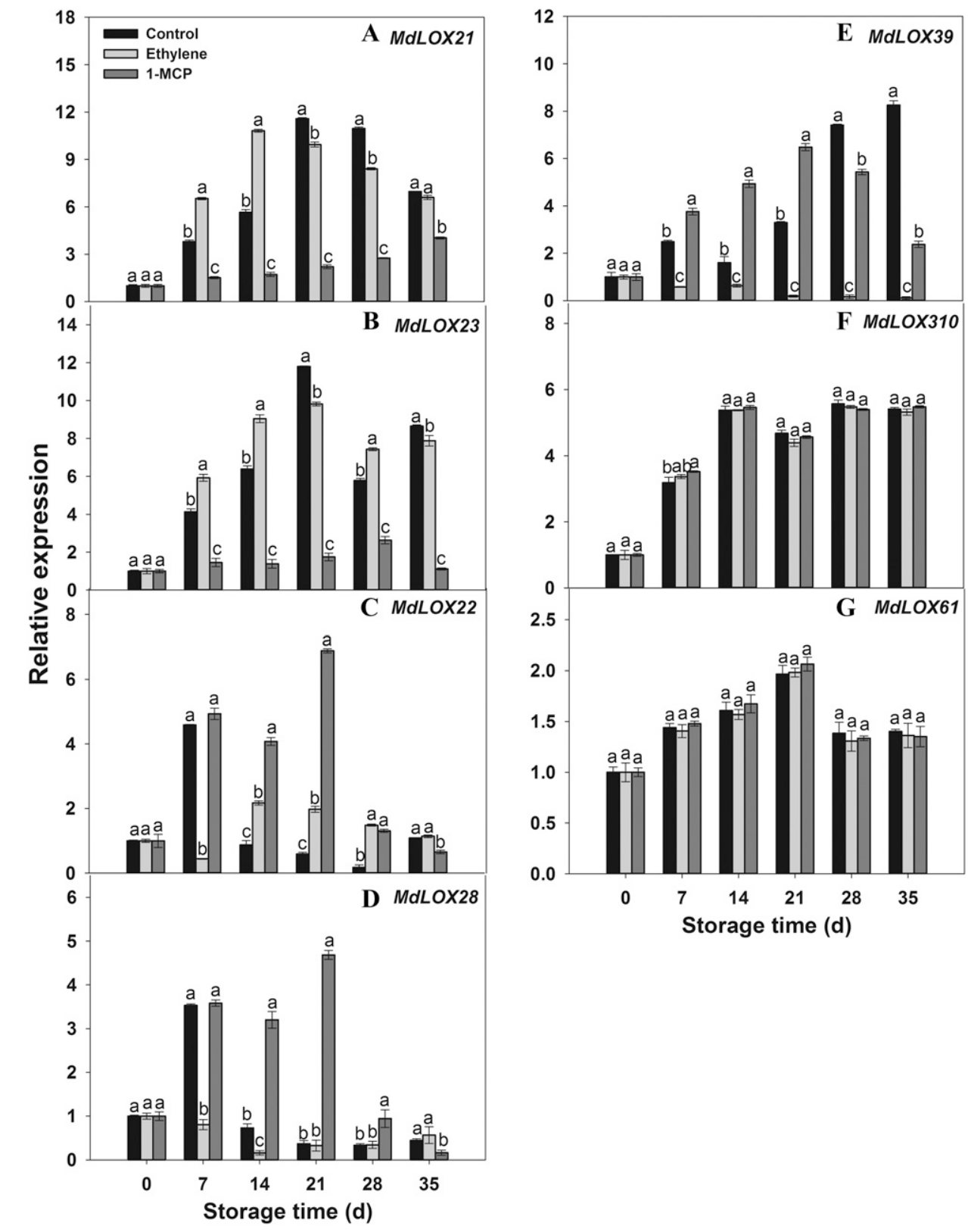

Fig. 2. Expression of $L O X$ genes in apple fruit during storage and after treatment with ethephon and 1-MCP. (A) Expression of $M d L O X 21,(\mathbf{B})$ expression of $M d L O X 23,(\mathbf{C})$ expression of $M d L O X 22$, (D) expression of $M d L O X 28,(\mathbf{E})$ expression of $M d L O X 39$, (F) expression of $M d L O X 310$, and (G) expression of $M d L O X 61$. Ethephon treatment was performed by immersing fruit into $0.2 \mathrm{mmol} \cdot \mathrm{L}^{-1}$ ethephon for $20 \mathrm{~min}$. 1 -MCP treatment was performed by enclosing fruit with $1 \mu \mathrm{L} \cdot \mathrm{L}^{-1} 1-\mathrm{MCP}$ for $24 \mathrm{~h}$. Untreated fruits served as controls. Mdactin was used as an internal standard for each gene. The values for each time point represent an average of three PCR runs for three biological replicates. Vertical bars represent SE. Columns with different letters at each time point indicate significant differences according to LSD at $P<0.05 . L O X=$ lipoxygenase; $\mathrm{MCP}=1$-methylcyclopropene; LSD = least significant difference. 
(Fig. 2). In the control fruit, the expression of MdLOX21 and MdLOX23 peaked at $21 \mathrm{~d}$ when the maximum accumulation of ethylene occurred (Fig. 2A and B). Their expression was upregulated by ethephon treatment only from 7 to $14 \mathrm{~d}$ whereas suppressed by 1-MCP treatment during storage compared with controls, indicating that they were to some extent positively regulated by ethylene.

The expression patterns of MdLOX22 and MdLOX28 were in accordance with the change of AOS activity in all groups (Fig. 2C and D). In the control fruit, their expression peaked at $7 \mathrm{~d}$ and then decreased from 7 to $28 \mathrm{~d}$. In the ethephon-treated fruit, the expression of MdLOX22 and MdLOX28 was downregulated at $7 \mathrm{~d}(P<0.01)$, and then it recovered to a level that was equal to or greater than the control level. In the 1-MCPtreated fruit, their expression peaked at $21 \mathrm{~d}$ and was obviously higher than the controls from 14 to $28 \mathrm{~d}$.

MdLOX39 exhibited an increased trend on ripening in the control fruit (Fig. 2E). The expression of MdLOX39 was downregulated by ethephon treatment throughout the entire storage period $(P<0.01)$, whereas it was upregulated by $1-\mathrm{MCP}$ treatment from 7 to $21 \mathrm{~d}(P<0.05)$ when the endogenous ethylene production was suppressed, suggesting some degree of negative effects of ethylene on its expression. Ethephon and 1-MCP treatment had no apparent effect on the expression of MdLOX310 and MdLOX61, indicating that they were not regulated by ethylene (Fig. 2F and G).

Generally, the expression profiles of the three AOS genes showed a similar trend as AOS activity in all groups. In the ethephon-treated fruit, the expression of MdAOSs was downregulated at $7 \mathrm{~d}$, and then it recovered after $14 \mathrm{~d}$ compared with the controls. In the 1-MCP-treated fruit, expression of MdAOS2 and $M d A O S 3$ was upregulated after $7 \mathrm{~d}$, and it peaked at 28 and $21 \mathrm{~d}$, respectively, which was later than the peak in the control fruit (Fig. 3A and B); the expression of MdAOS5 peaked at $21 \mathrm{~d}$, and it was only evidently upregulated from 14 to $28 \mathrm{~d}$ (Fig. 3C).

EXPRESSION PATTERNS OF MdJAZS DURING FRUIT RIPENING AND IN RESPONSE TO ETHEPHON AND 1-MCP TREATMENT. AmOng the five identified $J A Z$ genes, MdJAZ10 maintained more abundant transcript levels during ripening. The expression of $M d J A Z 9$ was upregulated by ethephon treatment throughout the entire storage period compared with the controls. In the 1-MCPtreated fruit, its expression was lower than the controls after $7 \mathrm{~d}$ (Fig. 4A). These data suggested that MdJAZ9 was positively regulated by ethylene in ripening apple fruit. The expression of MdJAZ10 gradually increased on ripening in the control fruit (Fig. 4B). In the ethephon-treated fruit, the expression of MdJAZ10 was reduced at $7 \mathrm{~d}$, and then it was upregulated and maintained at a higher level than the controls. By contrast, 1 -MCP treatment suppressed its expression after $14 \mathrm{~d}$. The expression of MdJAZ18 peaked at $21 \mathrm{~d}$ in all groups (Fig. 4C). It was upregulated by ethephon treatment only from 7 to $14 \mathrm{~d}$, and then it gradually decreased and was maintained at a lower level than the controls. 1-MCP treatment downregulated the expression of MdJAZ18 throughout the entire storage period. The expression of MdJAZ13 and MdJAZ14 was downregulated either by ethephon treatment or by 1-MCP treatment from 7 to $14 \mathrm{~d}$, and then it recovered to (MdJAZ13) or even became higher (MdJAZ14) than the control level (Fig. 4D and E).

\section{Discussion}

Ethylene is a relatively well-understood gaseous hormone that primarily regulates the ripening of climacteric fruit (Yang

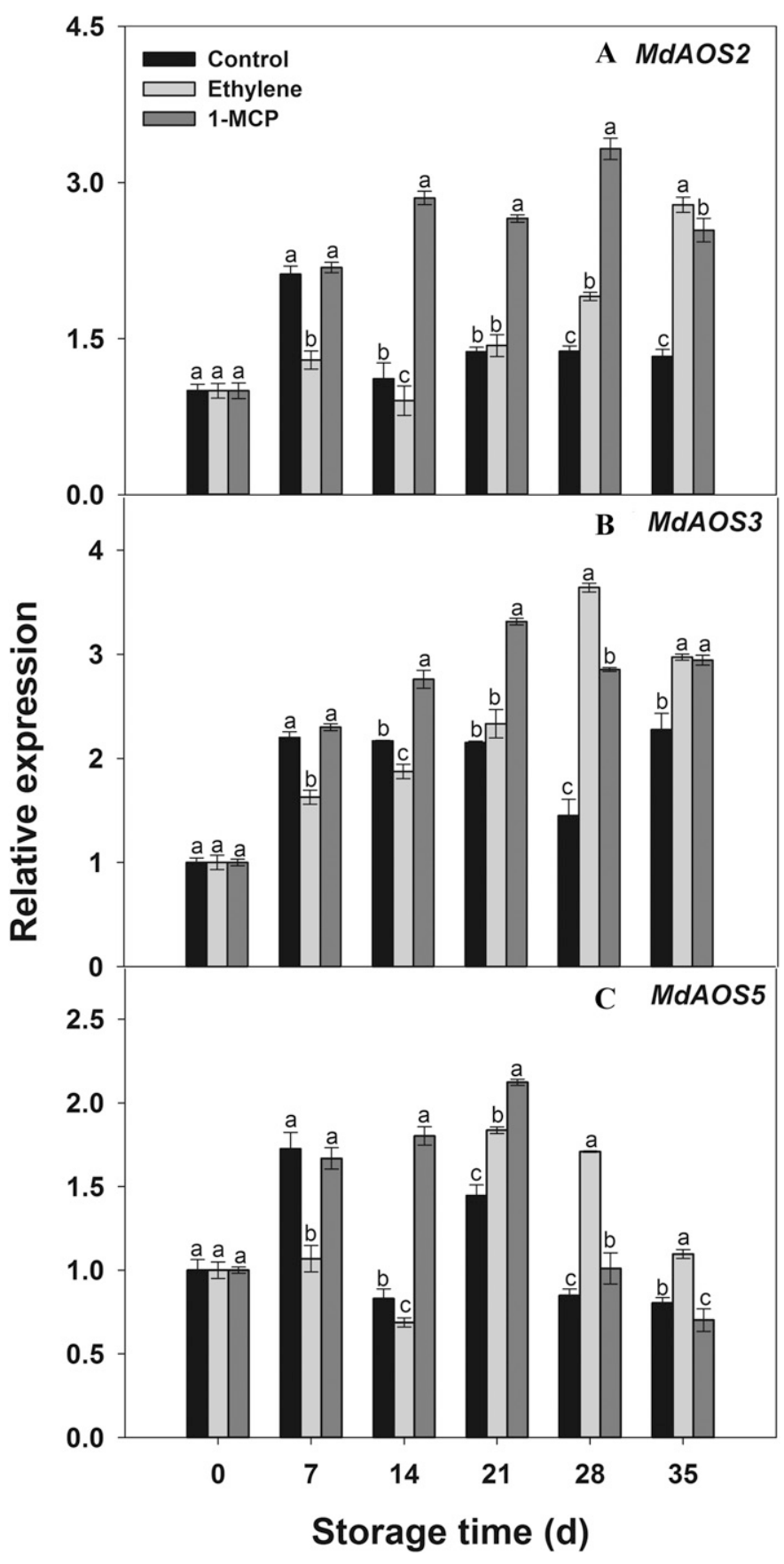

Fig. 3. Expression of $A O S$ genes in apple fruit during storage and after treatment with ethephon and 1-MCP. (A) Expression of MdAOS2, (B) expression of $M d A O S 3$, and (C) expression of MdAOS5. Ethephon treatment was performed by immersing fruit into $0.2 \mathrm{mmol} \cdot \mathrm{L}^{-1}$ ethephon for $20 \mathrm{~min}$. 1-MCP treatment was performed by enclosing fruit with $1 \mu \mathrm{L} \cdot \mathrm{L}^{-1} 1-\mathrm{MCP}$ for $24 \mathrm{~h}$. Untreated fruits served as controls. Mdactin was used as an internal standard for each gene. The values for each time point represent an average of three PCR runs for three biological replicates. Vertical bars represent SE. Columns with different letters at each time point indicate significant differences according to LSD at $P<0.05 . A O S=$ allene oxide synthase; $1-\mathrm{MCP}=1$-methylcyclopropene; $\mathrm{LSD}=$ least significant difference.

and Hoffman, 1984). In addition to ethylene, JAs also play a role in fruit maturation and ripening (Kondo, 2010). Previous studies have revealed that the levels of endogenous JAs were higher at harvest and then declined with the progression of ripening in climacteric fruit (Fan et al., 1998), which was in 


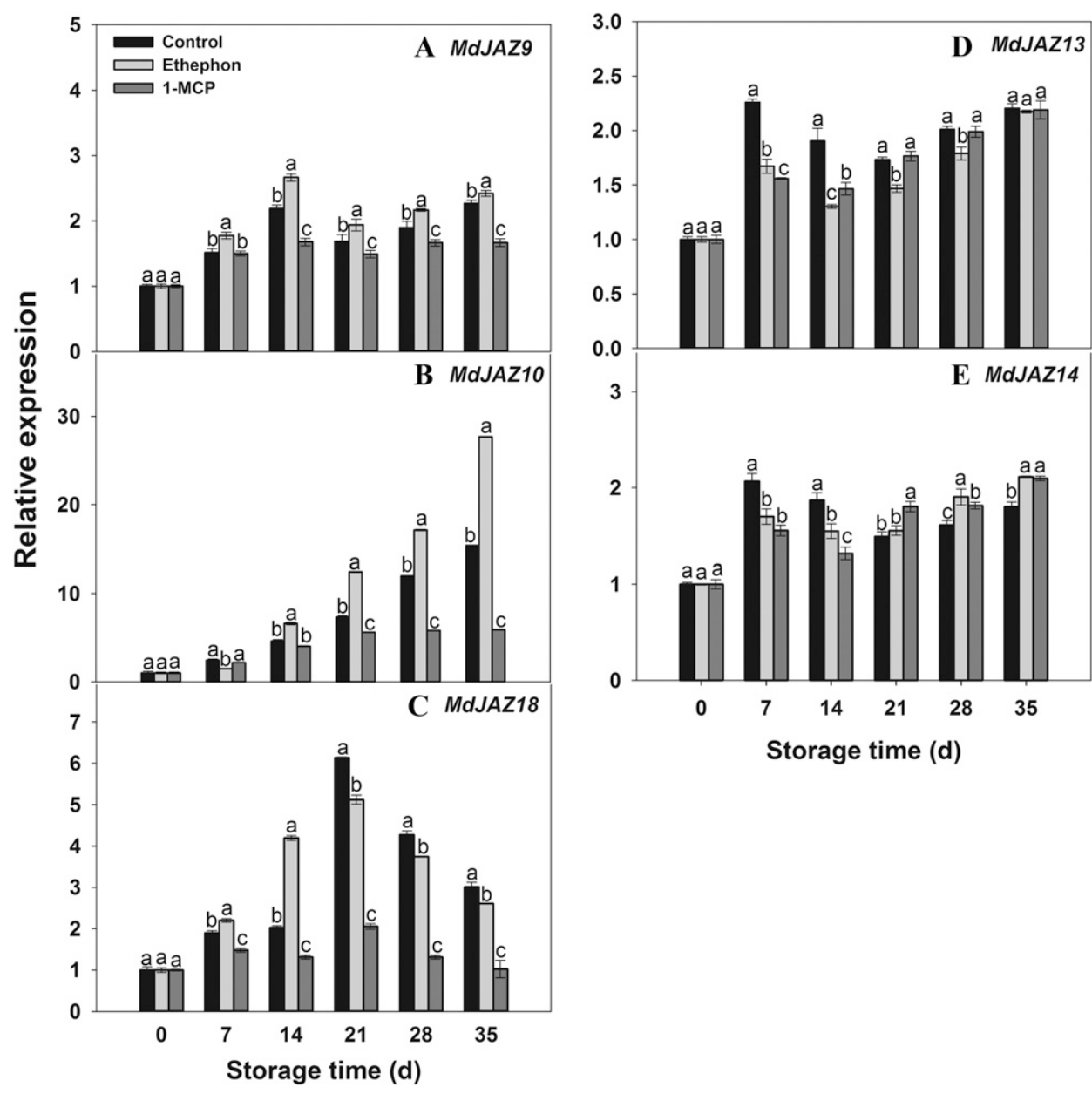

Fig. 4. Expression of $J A Z$ genes of apple fruit during storage and after treatment with ethephon and 1-MCP. (A) Expression of $M d J A Z 9,(\mathbf{B})$ expression of $M d J A Z 10,(\mathbf{C})$ expression of $M d J A Z 18,(\mathbf{D})$ expression of $M d J A Z 13$ and $(\mathbf{E})$ expression of $M d J A Z 14$. Ethephon treatment was performed by immersing fruit into $0.2 \mathrm{mmol} \cdot \mathrm{L}^{-1}$ ethephon for $20 \mathrm{~min}$. 1-MCP treatment was performed by enclosing fruit with $1 \mu \mathrm{L}^{-1} \mathrm{~L}^{-1} 1-\mathrm{MCP}$ for $24 \mathrm{~h}$. Untreated fruits served as controls. Mdactin was used as an internal standard for each gene. The values for each time point represent an average of three PCR runs for three biological replicates. Vertical bars represent SE. Columns with different letters at each time point indicate significant differences according to LSD at $P<0.05$. $J A Z=$ jasmonate ZIM-domain; 1-MCP = 1-methylcyclopropene; LSD = least significant difference.

agreement with our observation in the control fruit. JAs and ethylene act synergistically and antagonistically in the development and defense responses of several types of plants (Pieterse et al., 2009). In this study, the endogenous JA content was reduced after ethephon application, and then it gradually recovered toward senescence, suggesting the temporary antagonistic function of exogenous ethylene on JA biosynthesis during ripening. In general, AOS activity showed a similar pattern with the change of endogenous JA content in response to both treatments. The AOS activity was reduced at the early ripening stage after ethephon treatment in our research. However, studies on kiwifruit showed that the AOS activity was increased after the application of ethephon (Ding et al., 2003). The results that differ from those in the abovementioned studies may be due to the difference in the applied ethylene concentration and fruit type.

Seven LOX genes (MdLOX21, MdLOX22, MdLOX23, $M d L O X 28, M d L O X 39, M d L O X 310$, and MdLOX61) and three $A O S$ genes (MdAOS2, MdAOS3, and MdAOS5) were identified in ripening apple fruit. Ethephon treatment temporarily downregulated the expression of the three $M d A O S$ genes at the early ripening stage compared with controls, whereas 1-MCP treatment differentially upregulated their expression before $21 \mathrm{~d}$. These results suggested that ethylene might have a certain degree of negative impact on the expression of $M d A O S$ genes during ripening. Among the seven identified $L O X$ genes, expression patterns of $M d L O X 22$ and $M d L O X 28$ showed similar trends as that of three $M d A O S$ genes and the change of AOS activity in all groups, indicating that these two $L O X$ genes might be involved in the JA biosynthesis pathway during ripening. Conversely, expression patterns of $M d L O X 21$ and $M d L O X 23$ showed similar trends as the change of ethylene production in all groups, and their expression was positively regulated by ethylene during ripening. A similar result was obtained from kiwifruit AdLOX1 (Zhang et al., 2009). In addition to participating in JA biosynthesis, 13-LOX is also involved in other physiological processes, for example, the biosynthetic pathway of fruit volatile compounds (Feussner and Wasternack, 2002), which has been shown to be regulated by ethylene in apple fruit (Schaffer et al., 2007); therefore, we hypothesize that $M d L O X 21$ and MdLOX23 expression might be closely related to ethylene-dependent ripening processes. Schiller et al. (2015) observed that MdLOX39 contributed to aldehyde production in immature apple fruit on cell disruption but not during climacteric ripening. Our research showed that the expression of MdLOX39 was negatively regulated by ethylene during climacteric ripening. Further study is needed to clarify the role of MdLOX39 in ripening apple fruit. MdLOX310 and $M d L O X 61$ were not regulated by ethylene, whereas their expression changed considerably on fruit ripening, suggesting that they might be regulated by other ripening-related factors.

JAZ transcriptional repressors have been reported as key regulators of JA hormonal response in arabidopsis (Thines et al., 2007). Five $J A Z$ genes (MdJAZ9, MdJAZ10, MdJAZ13, $M d J A Z 14$, and $M d J A Z 18$ ) were identified in apple fruit after harvest. The expression of MdJAZ9, MdJAZ10, and MdJAZ18 was positively regulated by ethylene during fruit ripening. However, the expression of $M d J A Z 13$ and $M d J A Z 14$ was downregulated either by ethephon treatment or by 1-MCP treatment at an earlier ripening stage. Because ripening is a complex process involving many physiological changes and the dynamic interplay between different phytohormones (McAtee et al., 2013), we hypothesize that MdJAZ13 and $M d J A Z 14$ might be regulated by other JA-related factors. It has been confirmed that ethylene regulates ripening-related genes 
through a transcriptional cascade that comprises primary [ethylene-insensitive 3 and ethylene-insensitive 3 like (EIN3/ EIL)] and secondary response factors [ethylene response factors (ERFs)] (Liu et al., 2015). Zhu et al. (2011) observed that EIN3/EIL1 was the unique class of JAZ-targeted TFs that integrated ethylene and JA signaling in plant development and defense against necrotrophic pathogens. However, whether there is innate cross talk between JA and ethylene inside ripening apple fruit is largely unknown. Among all of the genes in our study, only the expression profile of $M d J A Z 10$ was in accordance and significantly correlated with that of MdEILs and MdERF1 on ripening (Supplemental Fig. 1; Supplemental Table 2), indicating that $M d J A Z 10$ might be the candidate gene that interacts with the ethylene signaling pathway during fruit ripening. A simplified model of the regulatory role of ethylene on JA biosynthesis and signaling pathway based on the observations and speculation from this study was shown in Supplemental Fig. 2.

Our data confirmed the hypothesis that ethylene might regulate the JA biosynthesis and signaling pathway during the ripening of apple fruit by applying 1-MCP and ethephon. Understanding the expression patterns of key genes involved in JA biosynthesis and signaling pathway in response to ethylene may help to decode the complex mechanisms of cross talk between the ethylene and JA signal transduction pathways during fruit ripening and senescence.

\section{Conclusions}

We first report that the JA biosynthesis and signaling pathway were differentially regulated by ethylene signaling during ripening of the apple fruit. Endogenous JA content, AOS activity, and expression of MdAOSs were reduced by ethephon treatment at the early ripening stage, whereas they were promoted by $1-\mathrm{MCP}$ treatment at the late ripening stage. The expression of $M d L O X 22$ and $M d L O X 28$ was in accordance with the change of AOS activity in all groups, indicating that they might be involved in the JA biosynthesis pathway on ripening. The expression of $M d L O X 21, M d L O X 23$, and three $M d J A Z$ genes (MdJAZ9, MdJAZ10, and $M d J A Z 18)$ was positively regulated by ethylene at varying degrees, whereas the expression of MdLOX39 was negatively regulated by it during ripening. The expression of MdLOX310 and MdLOX61 was not regulated by ethylene during ripening. The expression of $M d J A Z 13$ and $M d J A Z 14$ was downregulated by both treatments at the early ripening stage.

\section{Literature Cited}

Blanpied, G.D. and K.J. Silsby. 1992. Predicting harvest date windows for apples. Cornell Coop. Ext. Info. Bul. 221.

Deng, A., W. Tan, S. He, W. Liu, T. Nan, Z. Li, B. Wang, and Q.X. Li. 2008. Monoclonal antibody-based enzyme linked immunosorbent assay for the analysis of jasmonates in plants. J. Integr. Plant Biol. 50:1046-1052.

Ding, J., K. Chen, W. Xu, and C. Xu. 2003. Regulation of 1-methylcyclopropene on the ripening and softening of postharvest kiwifruit. Yuan Yi Xue Bao 30:277-280 (in Chinese with English abstract).

Ellis, C. and J.G. Turner. 2001. The Arabidopsis mutant cev1 has constitutively active jasmonate and ethylene signal pathways and enhanced resistance to pathogens. Plant Cell 13:1025-1033.

Fan, X., J.P. Mattheis, and J.K. Fellman. 1998. A role for jasmonate in climacteric fruit ripening. Planta 204:444-449.
Feussner, I. and C. Wasternack. 2002. The lipoxygenase pathway. Annu. Rev. Plant Biol. 3:275-297.

Guardo, D.M., A. Tadiello, B. Farneti, G. Lorenz, D. Masuero, U. Vrhovsek, G. Costa, R. Velasco, and F. Costa. 2013. A multidisciplinary approach providing new insight into fruit flesh browning physiology in apple (Malus $\times$ domestica Borkh.). PLoS One 8: e78004.

Katsir, L., H.S. Chung, A.J.K. Koo, and G.A. Howe. 2008. Jasmonate signaling: A conserved mechanism of hormone sensing. Curr. Opin. Plant Biol. 11:428-435.

Kondo, S. 2010. Roles of jasmonates in fruit ripening and environmental stress. Acta Hort. 884:711-716.

Kondo, S., S. Meemak, Y. Ban, T. Moriguchi, and T. Harada. 2009. Effects of auxin and jasmonates on 1-aminocyclopropane1-carboxylate (ACC) synthase and ACC oxidase gene expression during ripening of apple fruit. Postharvest Biol. Technol. 51:281284.

Kondo, S., S. Setha, D.R. Rudell, D.A. Buchanan, and J.P. Mattheis. 2005. Aroma volatile biosynthesis in apple affected by 1-MCP and methyl jasmonate. Postharvest Biol. Technol. 36:61-68.

Kondo, S., H. Yamada, and S. Setha. 2007. Effect of jasmonates differed at fruit ripening stages on 1-aminocyclopropane-1-carboxylate (ACC) synthase and ACC oxidase gene expression in pears. J. Amer. Soc. Hort. Sci. 132:120-125.

Laudert, D. and E.W. Weiler. 1998. Allene oxide synthase: A major control point in Arabidopsis thaliana octadecanoid signalling. Plant J. 15:675-684.

Li, X., X. Yin, H. Wang, J. Li, C. Guo, H. Gao, Y. Zheng, C. Fan, and X. Wang. 2015. Genome-wide identification and analysis of the apple (Malus $\times$ domestica Borkh.) TIFY gene family. Tree Genet. Genomes 11:1-13.

Liu, M., J. Pirrello, C. Chervin, J.P. Roustan, and M. Bouzayen. 2015. Ethylene control of fruit ripening: Revisiting the complex network of transcriptional regulation. Plant Physiol. 169:2380-2390.

Livak, K.J. and T.D. Schmittgen. 2001. Analysis of relative gene expression data using real-time quantitative PCR and the $2^{-\Delta \Delta \mathrm{Ct}}$ method. Methods 25:402-408.

Lv, J., J. Rao, F. Johnson, S. Shin, and Y. Zhu. 2015. Genome-wide identification of jasmonate biosynthetic genes and characterization of their expression profiles during apple (Malus $\times$ domestica) fruit maturation. Plant Growth Regulat. 75:355-364.

McAtee, P., S. Karim, R. Schaffer, and K. David. 2013. A dynamic interplay between phytohormones is required for fruit development, maturation, and ripening. Front. Plant Sci. 4:1-7.

Pérez, A.G., C. Sanz, D.G. Richardson, and J.M. Olías. 1993. Methyl jasmonate vapor promotes $\beta$-carotene synthesis and chlorophyll degradation in 'Golden Delicious' apple peel. J. Plant Growth Regul. 12:163-167.

Pieterse, C.M.J., A. Leon-Reyes, S. van der Ent, and S.C.M. Van Wees. 2009. Networking by small-molecule hormones in plant immunity. Nat. Chem. Biol. 5:308-316.

Rudell, D.R. and J.P. Mattheis. 2008. Synergism exists between ethylene and methyl jasmonate in artificial light induced pigment enhancement of 'Fuji' apple fruit peel. Postharvest Biol. Technol. 47:136-140.

Ruiz, K.B., L. Trainotti, C. Bonghi, V. Ziosi, G. Costa, and P. Torrigiani. 2013. Early methyl jasmonate application to peach delays fruit/seed development by altering the expression of multiple hormone-related genes. J. Plant Growth Regul. 32:852864.

Schaffer, R.J., E.N. Friel, E.J.F. Souleyre, K. Bolitho, K. Thodey, S. Ledger, J.H. Bowen, J.H. Ma, B. Nain, D. Cohen, A.P. Gleave, R.N. Crowhurst, B.J. Janssen, J.L. Yao, and R.D. Newcomb. 2007. A genomics approach reveals that aroma production in apple is controlled by ethylene predominantly at the final step in each biosynthetic pathway. Plant Physiol. 144:1899-1912.

Schiller, D., C. Contreras, J. Vogt, F. Dunemann, B. Defilippi, R. Beaudry, and W. Schwab. 2015. A dual positional specific lipoxygenase 
functions in the generation of flavor compounds during climacteric ripening of apple. Hort. Res. 2:1-13.

Song, S., T. Qi, C. Wasternack, and D. Xie. 2014. Jasmonate signaling and crosstalk with gibberellin and ethylene. Curr. Opin. Plant Biol. 21:112-119.

Thines, B., L. Katsir, M. Melotto, Y. Niu, A. Mandaokar, G. Liu, K. Nomura, S.Y. He, G.A. Howe, and J. Browse. 2007. JAZ repressor proteins are targets of the $\mathrm{SCF}^{\mathrm{CO} 11}$ complex during jasmonate signalling. Nature 448:661-665.

Tuominen, H., K. Overmyer, M. Keinänen, H. Kollist, and J. Kangasjärvi. 2004. Mutual antagonism of ethylene and jasmonic acid regulates ozone-induced spreading cell death in Arabidopsis. Plant J. 39:59-69.

Wasternack, C. 2007. Jasmonates: An update on biosynthesis, signal transduction and action in plant stress response, growth and development. Ann. Bot. 100:681-697.
Watkins, C.B. 2006. The use of 1-methylcyclopropene (1-MCP) on fruits and vegetables. Biotechnol. Adv. 24:389-409.

Yang, S.F. and N.E. Hoffman. 1984. Ethylene biosynthesis and its regulation in higher plants. Annu. Rev. Plant Physiol. 35:155-189.

Zhang, B., X. Yin, X. Li, S. Yang, I. Ferguson, and K. Chen. 2009. Lipoxygenase gene expression in ripening kiwifruit in relation to ethylene and aroma production. J. Agr. Food Chem. 57:28752881.

Zhu, Z., F. An, Y. Feng, P. Li, L. Xue, M. A, Z. Jiang, J.M. Kim, T.K. To, W. Li, X. Zhang, Q. Yu, Z. Dong, W.Q. Chen, M. Seki, J.M. Zhou, and H. Guo. 2011. Derepression of ethylene-stabilized transcription factors (EIN3/EIL1) mediates jasmonate and ethylene signaling synergy in Arabidopsis. Proc. Natl. Acad. Sci. USA 108:12539-12544.

Zimmerman, D.C. and B.A. Vick. 1970. Hydroperoxide isomerase: A new enzyme of lipid metabolism. Plant Physiol. 46:445-453. 

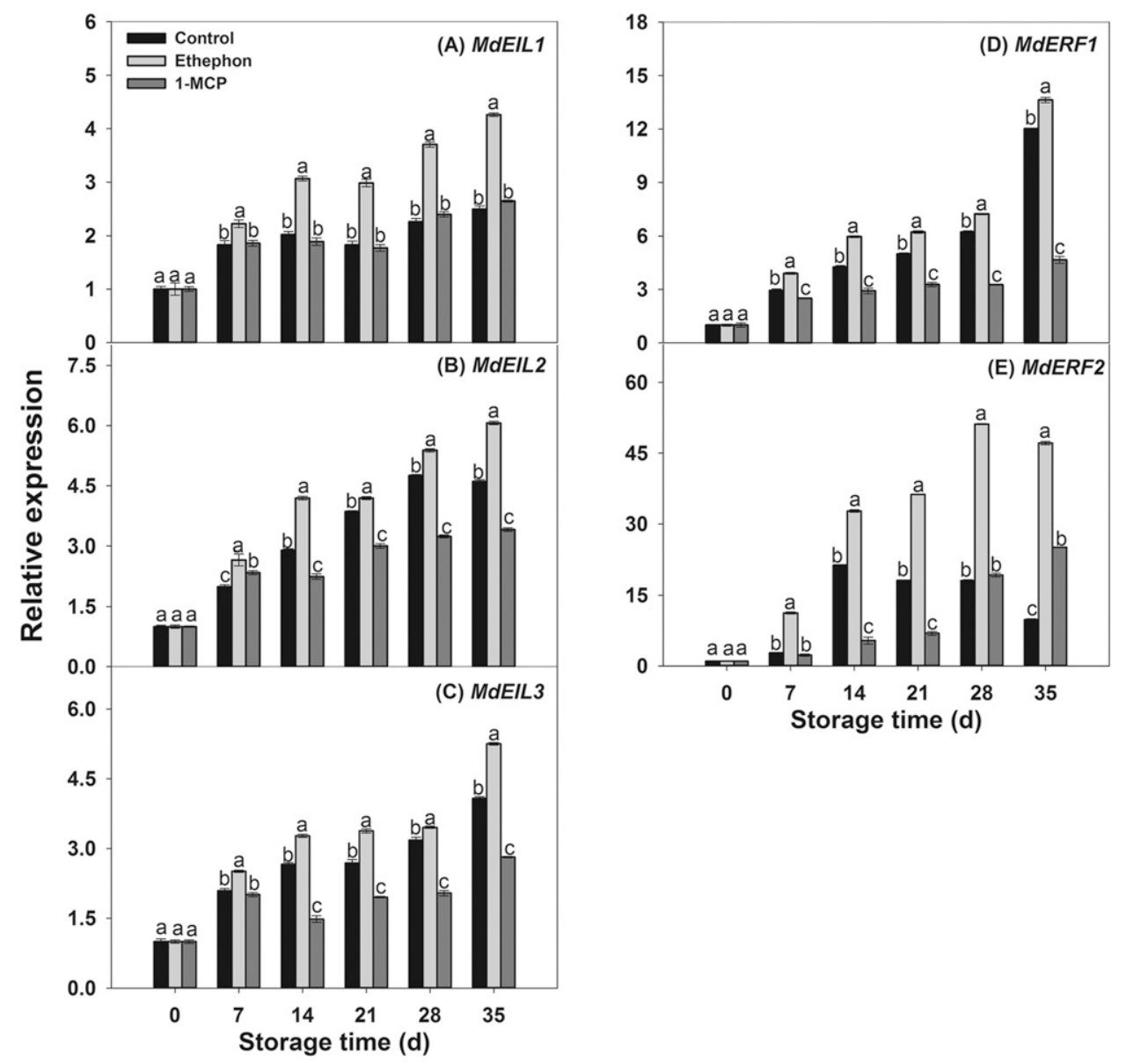

Supplemental Fig. 1. Expression of $E I L$ and $E R F$ genes of apple fruit during storage and after treatment with ethephon and 1-MCP. (A) Expression of MdEIL1, (B) expression of MdEIL2, (C) expression of $M d E I L 3$, (D) expression of $M d E R F 1$, and (E) expression of MdERF2. Ethephon treatment was performed by immersing fruit into $0.2 \mathrm{mmol} \cdot \mathrm{L}^{-1}$ ethephon for $20 \mathrm{~min}$. 1-MCP treatment was performed by enclosing fruit with $1 \mu \mathrm{L} \cdot \mathrm{L}^{-1} 1-\mathrm{MCP}$ for $24 \mathrm{~h}$. Untreated fruits served as controls Mdactin was used as an internal standard for each gene. The values for each time point represent an average of three PCR runs for three biological replicates. Vertical bars represent SE. Columns with different letters at each time point indicate significant differences according to LSD test at $P<0.05$. EIL $=$ ethyleneinsensitive 3 like; $E R F=$ ethylene response factor; 1-MCP = 1-methylcyclopropene; LSD = least significant difference.

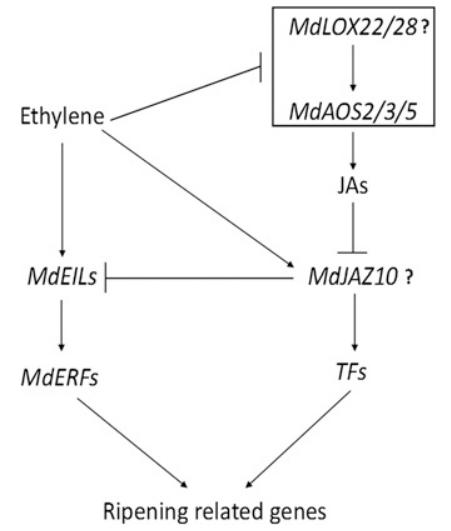

Supplemental Fig. 2. The proposed model of the regulatory role of ethylene on JA biosynthesis and signaling pathway in ripening apple fruit. Ethylene might affect JA biosynthesis by negative regulation of the expression of $L O X$ and $A O S$ genes. Active forms of JAs trigger the degradation of JAZ proteins that repress TFs involved in the expression of ripening-related events. JAZ proteins might directly interact with EIN3/EIL1 TFs. The degradation of JAZ proteins might induce the activity of EIN3/EIL1 and the expression of subsequent downstream $E R F$ for the induction of ripening-related events. Arrows indicate positive regulation, blunt ends indicate negative regulation, and question marks indicate the component still needs further verification. $\mathrm{JA}=$ jasmonate; $L O X=$ lipoxygenase; $A O S=$ allene oxide synthase; JAZ = jasmonate ZIM-domain; $\mathrm{TF}=$ transcription factor; EIN3/EIL1 = ethylene-insensitive 3 and ethylene-insensitive 3 like. 
Supplemental Table 1. Primer sequences.

\begin{tabular}{|c|c|c|}
\hline Gene name ${ }^{z}$ & Forward primer & Reverse primer \\
\hline$M d L O X 21$ & AACTGAAGATCCATCTGAC & TCACCAATATACTCCTCATC \\
\hline$M d L O X 22$ & GAAGGGGTATGGGCACAA & ACATTAACAGTGCCGTTTGG \\
\hline$M d L O X 23$ & GAGTTCAGTTCTGTTGCTTACG & CACTCCTTAATGGCATCCC \\
\hline$M d L O X 25$ & GCTGTAGGAGGAGTGGGA & CCTTCCAGCCGGAAATTAA \\
\hline$M d L O X 26$ & AACTAGATCCTGCTGTTTACG & GCTTCCTGTAGCTGATCTAAT \\
\hline$M d L O X 27$ & TGCTCACAACTTCTGCCACAAAC & TGGCTCCAGTCAGTGCTGAATTA \\
\hline$M d L O X 31$ & CTCGTCAAGCTCTGGTATTG & GCAGTTGAGGAAATGACTTGAG \\
\hline$M d L O X 32$ & CTTTTACCACACCTTCACTGGC & CTGTGAGGAATAAGAACAAGGA \\
\hline$M d L O X 33$ & CCAGTTTTAGATCCTTGGACC & AGGAGACCTCTGAAGTCAAATA \\
\hline$M d L O X 34$ & CAACGGAAGCTTCATCTTTTG & TACGTGCCTAGAGACGAGG \\
\hline$M d L O X 35$ & GCCTATTAAATGTTCСТCСТC & CCAAAATTAATCTCAAGGGATA \\
\hline$M d L O X 36$ & GAGGTTAATTCGAGACAATTAGG & GGAGATGAAGAAAATTGACAAGG \\
\hline$M d L O X 311$ & ATAGATTTTCAATGGAGATGAAG & TTAACCCTTG ACTGTTATACTA \\
\hline$M d L O X 41$ & AAGAGTTCTACTTGTTGGAGA & ATCTGAGATACAAAAGCAACG \\
\hline$M d L O X 61$ & GACTCATGCTTCCGTTGAAC & CAGGACTAAAAGAAGCCTCAA \\
\hline$M d L O X 62$ & ATGGATAGATTCTCGTGGTTG & CCATTGATTTCTTGCTCTAAC \\
\hline MdAOS1 & GACTACTTCTACAACCAAGGC & GGTGGTGTCAAAGAGAATGG \\
\hline MdAOS2 & ACATCTGCTTCTATTGTGTCC & GAGTTTACTCGACTTTTTCCG \\
\hline$M d A O S 3$ & CTACAAATCCAAGGTCGTCG & GGTAGGAAAGGATGCGGTA \\
\hline MdAOS4 & CCAACACTCCCAGATTTT C & CGCTATCATATTCGCATACA \\
\hline MdAOS5 & GAAATCTGTGG CTACGAAGC & ATCAGCTCTCTCGAAAAT CC \\
\hline$M d J A Z 3 / 4$ & GGGGATGGAAGAAAGGGTG & GCTTGCTTGAGTCCGATGC \\
\hline$M d J A Z 5 / 6$ & CCGCACCCTAATGAAACCC & CCAACATCACGAACGCACA \\
\hline MdEIL3 & AGCTAATAACTGTGCACAAGATTGG & CCCATTTAGCACTGACACAAACCAC \\
\hline$M d E R F 1$ & TCCAGACCGGTTCTTACTATTAT & CAGCATCCACAGGTACAAC \\
\hline$M d E R F 2$ & GCCAGCAGTAGCACTTC & CCAATAGTTGCTCGCCAAT \\
\hline Mdactin & TGACCGAATGAGCAAGGAAATTACT & TACTCAGCTTTGGCAATCCACATC \\
\hline
\end{tabular}

$\overline{\mathrm{z}} \mathrm{LOX}=$ lipoxygenase; AOS = allene oxide synthase; JAZ = jasmonate ZIM-domain; EIL = ethylene-insensitive 3 like; ERF = ethylene response factor.

Supplemental Table 2. Pearson correlation coefficients between the expression of jasmonate ZIM-domain (JAZ) genes and the expression of ethylene-insensitive 3 like $(E I L)$ and ethylene response factor $(E R F)$ genes involved in the ethylene signal pathway in apple fruit without any treatment during storage. ${ }^{\mathrm{z}}$

\begin{tabular}{|c|c|c|c|c|c|}
\hline & MdJAZ9 & MdJAZ10 & $M d J A Z 13$ & MdJAZ14 & $\overline{M d J A Z 18}$ \\
\hline$\overline{M d E I L 1}$ & 0.595 & $0.830 * y$ & 0.298 & -0.220 & 0.443 \\
\hline MdEIL2 & 0.451 & $0.917^{*}$ & -0.199 & -0.733 & 0.719 \\
\hline MdEIL3 & 0.686 & $0.907 *$ & 0.170 & -0.317 & 0.488 \\
\hline$M d E R F 2$ & 0.727 & 0.349 & -0.839 & -0.662 & 0.618 \\
\hline
\end{tabular}

${ }^{\mathrm{Z}}$ Pearson correlation coefficients were calculated using SAS statistical software (version 8.0; SAS Institute, Cary, NC).

y*,**Denoted significant correlations at the level of $P<0.05$ and $P<0.01$, respectively. 